\title{
ANÁLISIS DE LA MICOBIOTA EXISTENTE EN EL AMBIENTE INTERIOR DE LA MAPOTECA DEL ARCHIVO NACIONAL DE LA REPÚBLICA DE CUBA
}

\author{
(Analysis of mycobiota existing in the indoor environment in the map library \\ of the national archive of the republic of Cuba)
}

\author{
Alian Molina V. ${ }^{1 *}$ \& Sofía Borrego A. ${ }^{2}$ \\ 1,2Laboratorio de Conservación Preventiva, Archivo Nacional de la República de Cuba. \\ Compostela 906 esquina a Calle San Isidro, La Habana Vieja, La Habana, Cuba. \\ *Autor para correspondencia:alian@arnac.cu
}

RECIBIDO: 09 de enero de 2014 APROBAD0: 20 de marzo de 2014

LOS AUTORES DECLARAN NO TENER CONFLITCTO DE INTERESES.

Palabras clave: hongos filamentosos, calidad de ambiente interior, muestreo aerobiológico, patrimonio documental. Key words: aerobiological sampling, documentary heritage, filamentous fungi, indoor environmental quality.

\section{RESUMEN}

Actualmente el estudio de la concentración fúngica en ambientes interiores es de interés para los especialistas, pues los hongos pueden causar el biodeterioro de colecciones valiosas y resultan peligrosos para la salud humana ya que provocan alergias y otras enfermedades al hombre que está en contacto con ellos. El objetivo del trabajo fue evaluar microbiológicamente el ambiente de la Mapoteca del Archivo Nacional de la República de Cuba y el polvo depositado sobre el mobiliario que conserva los mapas y planos en ese local. El estudio se ejecutó en un mes lluvioso y el muestreo microbiológico del aire se realizó empleando un método de sedimentación mientras que el polvo se colectó por aspiración. Los hongos se aislaron en medios de cultivo adecuados y los análisis se realizaron por triplicado. La concentración fúngica detectada en el aire fue inferior a las 750 UFC. $\mathrm{m}^{-3}$, por lo que el local se consideró POCO CONTAMINADO mientras en el polvo fue del orden de $10^{5} \mathrm{UFC} . \mathrm{g}^{-1}$. Se detectaron los géneros Alternaria, Aspergillus, Chaetomium, Acremonium, Cladosporium, Curvularia, Humicola, Epicoccum, Penicillium, Pestalotia y Trichoderma. Algunas de las especies fúngicas detectadas en el aire del local poseen importancia para el biodeterioro y la salud humana. Se reportan nuevos registros para el Archivo Nacional de la República de Cuba consistente en tres géneros (Epicoccum Link, Humicola Traaen y Pestalotia Not.), cuatro especies de Aspergillus Nees ex Fr. (A. alliaceus
Thom y Church., A. auricomus (Gueren) Saito., A. ostianus Wehmer y A. chevalieri (Mangin) Thom y Church.), una especie de Cladosporium Link ex Fr. (C. caryigenum Ellis y Lang.), dos especies de Penicillium Link (P. canescens Sopp., P. janczewskii K.M. Zalessky) y una de Curvularia Boedijn (C. australiensis Manamgoda, Cai y Hyde).

\section{ABSTRACT}

Currently the study of indoor fungal concentration is of interest for specialists, because the fungi cause the biodeterioration of the valuable collections and they are dangerous to human health as they cause allergies and other diseases to man who is in touch with them. The objectives were to evaluate the microbiological environment of Map Library of National Archive of the Republic of Cuba and the dust deposited on the furniture that keeps the maps and plans in this local. The study was carried out in a rainy month, microbial air sampling was performed using a sedimentation method and the powder was collected by manual vacuum aspiration. The fungi were isolated in suitable culture media and analyzes were performed by triplicate. The fungal concentration was detected in the air below the 750 CFU.m ${ }^{-3}$, so that the place was considered slightly contaminated while the dust was around $10^{5} \mathrm{CFU} . \mathrm{g}^{-1}$. The fungal genera Alternaria, Aspergillus, Chaetomium, Acremonium, Cladosporium, Curvularia, Humicola, Epicoccum, Penicillium, Pestalotia and Trichoderma were detected. 
Some fungal species detected in the repository air have importance for human health and biodeterioration. New records to the National Archives of the Republic of Cuba are reported consists of three genera (Epicoccum Link, Humicola Traaen, Pestalotia Not.), four species of Aspergillus Nees ex Fr. (A. alliaceus Thom \& Church., A. auricomus (Gueren) Saito., $A$. ostianus Wehmer. and $A$. chevalieri (Mangin) Thom y Church), one species of Cladosporium Link ex Fr. (C. caryigenum Ellis \& Lang.), two species of Penicillium Link (P. canescens Sopp., P. janczewskii K.M. Zalessky) and one of Curvularia Boedijn (C. australiensis Manamgoda, Cai \& Hyde ).

\section{INTRODUCCIÓN}

En los ambientes exteriores e interiores se encuentra un gran número de partículas de diferente origen, forma y tamaño suspendidas en el aire, ellas constituyen el aerosol atmosférico. Se pueden clasificar de diferentes formas, teniendo en cuenta el origen (biológico, orgánico, inorgánico), la localización (marina, continental, rural, industrial, urbana) y el efecto que pueden causar sobre las superficies en que se depositan (químico, tóxico, patogénico, degradativo). Entre las partículas de origen biológico se encuentran bacterias, esporas fúngicas, algas, virus, protozoos, granos de polen, etc. (Mandrioli, 2002).

Dentro de los componentes del aerosol atmosférico, también se encuentra el polvo, el cual se deposita sobre documentos, libros y otros objetos; el mismo varía en cantidad y calidad en dependencia de la situación del edificio, de las actividades que ocurran en su interior, de la estación del año y del estado de conservación del inmueble (Maggi et al, 2000). El polvo sirve como fuente de nutrientes para algunos insectos y microoganismos, estos últimos además pueden ser transportados por las partículas de polvo hacia el interior de locales a través de los sistemas de ventilación y los visitantes (Nevalainen y Morawska, 2009). En archivos y museos su sedimetación puede crear un microambiente sobre las superficies de las colecciones impidiendo el flujo normal del aire sobre ellos, lo que facilita que las áreas superficiales absorban gran cantidad de humedad, favoreciendo la proliferación de plagas como los hongos filamentosos (Florian, 2004; Pasquarella et al., 2012). La colonización y el crecimiento sobre la superficie de los objetos que se encuentran en el interior de los locales por los hongos, también puede ser una importante fuente de contaminación del aire ya sea con sus propágulos 0 compuestos volátiles producto de su metabolismo (Nevalainen y Morawska, 2009; Cappitelli y Sorlini, 2010; Karbowska-Berent et al., 2011). Por todo ello, desde hace algunos años es de gran interés, el conocimiento de la contaminación microbiana en el ambiente interior de locales de archivos y bibliotecas debido a la importancia que tienen los microorganismos en general y los hongos en particular, en el biodeterioro de diferentes materiales (Michaelsen et al., 2010; Borrego et al., 2010; Borrego y Perdomo, 2012; Guiamet et al., 2012; Guiamet et al., 2013).

En condiciones ambientales apropiadas, la micobiota del aire puede coexistir con las colecciones de valor histórico en un ecosistema específico sin causar grandes daños. Sin embargo, al producirse un incremento de la temperatura y la humedad relativa, los hongos pueden tener efectos negativos sobre las colecciones (Borrego et al., 2010 a; Guiamet et al., 2011). De ahí que para definir la calidad del aire en ambientes interiores, es necesario el conocimiento de la concentración fúngica en el interior de los locales. Este aspecto reviste una mayor significación en países de clima tropical al encontrarse un elevado nivel de propágulos fúngicos en el aire. Esta condición unida a los elevados contenidos de humedad relativa en él, favorecen la deposición de éstos sobre los sustratos y por tanto su desarrollo, propiciándose así, el biodeterioro de los soportes tanto de origen orgánico como inorgánico (Guiamet et al., 2011).

Otras propiedades importantes de los hongos están relacionadas con sus potencialidades patogénicas, es decir, la capacidad que tienen muchas especies para provocar enfermedades al hombre que está en contacto con ellos (Huttunen et al., 2003; Anderson, 2003; Denning, 2006; Nevalainen y Morawska, 2009; Haleem y Mohan, 2012). En varias investigaciones realizadas en archivos y bibliotecas, se ha demostrado que estos agentes son la causa de muchas enfermedades profesionales y patologías situacionales (Florian, 2003; Valentín, 2010; Haleem y Mohan, 2012).

Teniendo en cuenta estos aspectos, nos propusimos en este trabajo evaluar la micobiota existente en el ambiente de la Mapoteca del Archivo 
Nacional de la República de Cuba (ARNAC) y en el polvo depositado sobre el mobiliario que conserva los mapas y planos en ese local.

\section{MATERIALES Y MÉTODOS}

\section{Características del depósito estudiado}

La Mapoteca del ARNAC es un depósito que se caracteriza por su gran tamaño; sus dimensiones son las siguientes (largo $x$ ancho $x$ altura): $15.2 \times 6.2 \times 2.5$ $\mathrm{m}$. El local cuenta con un total de 195 metros lineales de mapas planos y privilegios de invención que están en su mayoría elaborados sobre papel de distintos tipos (lo que se conoce como papel para mapas desde el siglo XVIII (papel de fibras largas, resistente, grueso), papel vegetal de diferentes grosores, papel industrial ordinario de diferentes grosores, papel antiguo o de trapo propio del siglo XVIII, cartón ordinario del siglo XX, etc.), aunque hay algunos mapas que en lugar de papel poseen como soporte tejidos, tales como algodón, lino y seda.

\section{Medición de temperatura (T) y humedad relativa} (HR) en el momento del muestreo microbiológico

Aunque es habitual desde hace varios años la medición de la temperatura y humedad relativa dos veces al día en todos los depósitos del archivo (10:00 a.m. y 3:00 p.m.), durante la colecta de las muestras microbiológicas, se realizaron las mediciones en cada punto empleando un termohigrómetro digital (tabla I).

\section{Muestreo microbiológico del aire}

El trabajo experimental se realizó el 6 de junio del 2010 y consistió en un solo muestreo con tres réplicas por puntos. Se llevó a cabo siguiendo el método de sedimentación descrito por Omeliansky
(Bogomolova y Kirtsideli, 2009; Borrego et al., 2010; 2010 a). Para ello, placas Petri de $110 \mathrm{~mm}$ de diámetro que contenían Agar Malta (AM) suplementado con $\mathrm{NaCl}(7.5 \%)$ (Rojas et al., 2002), se colocaron abiertas aproximadamente a 2 metros sobre el nivel del suelo y se expusieron durante 5 minutos. Se muestrearon 5 puntos por triplicado, el número de puntos de muestreo se estableció de acuerdo con Sánchis (2002).

\section{Determinación de las unidades formadoras de colonias (UFC) por $\mathrm{m}^{-3} \mathrm{de}$ aire}

Las placas se incubaron invertidas durante 5 días a $30^{\circ} \mathrm{C}$. Concluida la incubación, se realizó el conteo de unidades formadoras de colonias y la concentración fúngica expresada en unidades formadoras de colonias por $\mathrm{m}^{3}$ de aire (UFC. $\mathrm{m}^{-3}$ ) se calculó según la ecuación descrita por Omeliansky (Bogomolova y Kirtsideli, 2009; Borrego et al., 2010, 2010a; Borrego et al., 2012):

$$
\mathrm{N}=5 \mathrm{a} \cdot 10^{4}(\mathrm{bt})^{-1}
$$

Donde: $\mathbf{N}$ - concentración microbiana en UFC. $\mathrm{m}^{-3}$, anúmero de colonias por placa Petri, $\mathbf{b}$ - radio de la placa Petri al cuadrado x ठ $\left(\mathrm{cm}^{2}\right), \mathbf{t}$-tiempo de exposición en minutos.

\section{Muestreo microbiológico del polvo}

La toma de la muestra se realizó una semana después de haber realizado la última limpieza. Para ello se utilizó una aspiradora eléctrica que permitió colectar el polvo depositado sobre los planeros 0 planotecas (mobiliario de gavetas grandes donde se conservan de forma abierta planos, mapas y otros documentos de gran formato). Posteriormente a $0.1 \mathrm{~g}$

Tabla I. Concentración fúngica detectada en el aire de la Mapoteca.

\begin{tabular}{|l|l|l|l|}
\hline Concentración & Hongos(UFC. $\mathbf{m}^{-3}$ ) & $\left.\mathbf{T}^{0} \mathbf{C}\right)$ & HR(\%) \\
\hline Máxima & $909.0^{\mathrm{a}}$ & 29.6 & 68.0 \\
Mínima & $363.0^{\mathrm{a}}$ & 29.4 & 67.0 \\
Media & $599.6^{\mathrm{b}}$ & $29.5^{\mathrm{c}}$ & $67.5^{\mathrm{c}}$ \\
\hline
\end{tabular}

T temperatura. HR humedad relativa. ${ }^{\text {a }}$ Tiene en cuenta el valor promedio de la concentración fúngica en cada punto

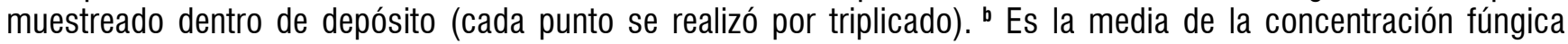
teniendo en cuenta las 15 placas utilizada. ${ }^{\circ}$ Representa la media de cinco mediciones que se corresponden con los puntos de muestreo microbiológico. 
Análisis de la micobiota existente en el ambiente interior de la mapoteca - A. Molina et al

del polvo colectado se le adicionó $1 \mathrm{ml}$ de agua destilada estéril, se agitó bien la muestra a intervalos de 15 minutos durante un tiempo total de 45 minutos. Luego se hicieron diluciones seriadas que se sembraron a profundidad en placas de $110 \mathrm{~mm}$ en un medio de cultivo de composición similar al descrito anteriormente. Las placas se incubaron invertidas durante 5 días a $30^{\circ} \mathrm{C}$.

\section{Determinación de UFC por gramo de polvo}

Concluida la incubación, se realizó el conteo de unidades formadoras de colonias y se determinó la concentración microbiana expresada en unidades formadoras de colonias por $g$ de polvo aspirado $\left(U F C . g^{-1}\right)$.

$$
\text { UFC. } \mathrm{g}^{-1}=\frac{\begin{array}{l}
\text { (Número de UFC totales obtenidas } \\
\mathrm{x} \text { dilución) } \mathrm{x} 1 \mathrm{~g} \text { de polvo }
\end{array}}{0.1 \mathrm{~g} \text { de polvo colectado }}
$$

\section{Identificación de las cepas aisladas}

Se realizó el aislamiento y la purificación de las diferentes colonias de microorganismos que crecieron en las placas utilizadas. Para la identificación taxonómica se tuvieron en cuenta las características culturales y morfológicas observadas al microscopio y estereoscopio según resultó conveniente. Dichas características se determinaron a partir de colonias de cada aislado obtenidas por inoculación en el medio AM. Para la observación de las características morfológicas se montaron microcultivos según la técnica de Casadesús y Rojas (1981), así como preparaciones en fresco, semipermanentes con lactofenol. Para la observación de estructuras hialinas se utilizó lactofenol-azul algodón (Johnston y Booth, 1983) 0 lactofenol-fushina (Klich y Pitt, 1994). Las observaciones se realizaron en un microscopio biológico trinocular de campo claro con cámara digital acoplada. La identificación taxonómica hasta nivel de género se realizó de acuerdo con los criterios de Kendrick y Carmichael (1973) y Barnett y Hunter (2003).

Para la identificación de las especies ubicadas en los géneros Aspergillus y Penicillium se siguieron los procedimientos sugeridos por Klich y Pitt (1994) y Pitt (2000). Estas metodologías están basadas primeramente en los caracteres morfológicos y en características fisiológicas como son las relaciones agua - temperatura, la pigmentación y el grado de desarrollo de las colonias en ciertos medios. Estas características se determinaron a los 7 días de inoculadas las cepas en los medios Agar Czapek Extracto de Levadura, incubadas a $5^{\circ} \mathrm{C}, 25^{\circ} \mathrm{C}$ y $37^{\circ}$ C, y en los medios Agar Extracto de Malta y Agar Czapek Extracto de Levadura con 20\% Sacarosa e incubadas a $25^{\circ} \mathrm{C}$ para Aspergillus y además Agar Nitrato de Glicerol $25 \%$ a $25^{\circ} \mathrm{C}$ para Penicillium.

Las especies ubicadas en los géneros Cladosporium y Curvularia se sembraron en medio Agar Extracto de Malta y se les efectuaron microcultivos para observar las características morfológicas. Para Cladosporium se siguieron los criterios de Castañeda (1996) y en el caso de las especies ubicadas en el género Curvularia se identificaron según lo reportado por Mena (2004) y Manamgoda et al. (2012). Para la identificación de las especies de este género se observaron al microscopio de campo claro las siguientes características: distribución en los conidióforos, forma, color, textura, número de septos y dimensiones. En todos los casos se realizaron al menos 20 observaciones distribuidas en varios campos de visión, en preparaciones tanto de la parte joven como de la zona madura de la colonia.

\section{Determinación de la densidad relativa (DR) de los taxa en el aire y en el polvo}

Este análisis se realizó de acuerdo a Smith (1980) donde:

\section{Número de colonias de \\ $D R=\quad$ un género o especie $\quad \times 100$ \\ Número total de colonias \\ de los géneros o especies \\ RESULTADOS Y DISCUSIONES}

\section{Grado de contaminación microbiana ambiental}

La concentración fúngica estimada en el aire interior de la Mapoteca se muestra en la tabla I, al analizar los promedios de cada punto muestreado (por triplicado) se puede constatar que el valor máximo alcanzado fue de 909 UFC. $\mathrm{m}^{-3}$ mientras que la media (de 15 placas) fue de 599.6 UFC. m $^{-3}$. Para determinar el grado de contaminación de un ambiente interior Omeliansky propuso una escala (Bogomolova y Kirtsideli, 2009; Borrego et al., 2010, 2010a; Borrego et al., 2012) y estableció que: concentraciones microbianas $d » 500$ UFC. $\mathrm{m}^{-3}$ es propio de un ambiente no contaminado, si la concentración microbiana está 501 - 750 UFC. $\mathrm{m}^{-3}$ es poco contaminado, si se encuentra 
Análisis de la micobiota existente en el ambiente interior de la mapoteca - A. Molina et al

entre 751 - 1000 UFC. $\mathrm{m}^{-3}$ es ligeramente contaminado, si está entre 1001 - 1500 UFC.m ${ }^{-3}$ es un ambiente contaminado y si es e»1501 UFC. $\mathrm{m}^{-3}$ es altamente contaminado.

Cuando se comparan los valores obtenidos con la escala que propuso Omeliansky para evaluar el grado de contaminación del aire, se puede concluir que al ser la concentración media inferior a 750 UFC. $\mathrm{m}^{-3}$, el ambiente del local se considera poco contaminado. Aunque desde hace algunos años grupos multidisciplinarios de científicos han dedicado esfuerzos y tiempo en los estudios de calidad del aire interior de locales por el riesgo potencial que implica la presencia de hongos en ambientes interiores para la salud humana y la integridad del patrimonio, en la actualidad no existe un consenso internacional en cuanto a regulaciones que establezcan valores límites que permitan clasificar a un ambiente interior de contaminado o no (Rojas y Aira; 2012). No obstante, en la literatura se encuentran disponibles algunos reportes más recientes para niveles de contaminación fúngica en ambientes interiores desarrollados por otros autores. La Organización Mundial de la Salud (OMS) plantea que un ambiente interior con una concentración mayor a 1000 UFC. $\mathrm{m}^{-3}$ se considera contaminado (Nevalainen y Morawska, 2009). En Brasil se considera que un ambiente interior con más de 700 UFC. $\mathrm{m}^{-3}$ de hongos, está contaminado (Radler de Aquino y de Góes, 2000). En Estados Unidos, American Conference of Industrial Hygienists y US Public Health Service proponen que 200 UFC.m ${ }^{-3}$ resulta un valor preocupante para bioaerosoles fúngicos (InspectaPedia, 2009), mientras que la Unión Europea estableció desde 1998 en las normas SBM (SBM, 2003), que 500 UFC. $\mathrm{m}^{-3}$ es el valor límite a partir del cual se considera un ambiente interior altamente contaminado. Asimismo, dentro de la Unión Europea otros autores plantean que 150 UFC. $\mathrm{m}^{-3}$ debe ser el límite de hongos permisibles para que el ambiente interior de locales en instituciones patrimoniales se considere de calidad (Cappitelli et al. 2009). Como se puede constatar en lo antes expuesto, los criterios con respecto a esta problemática son disímiles según, regiones, países, organizaciones o autores. Consideramos que la normalización de la calidad microbiológica ambiental de interiores de forma general debe estar en concordancia con el tipo de edificación, su función social y la zona climática o país donde se encuentra. Por tanto la aceptación de criterios de peso para su normalización requiere de la realización toda una serie de estudios microbiológicos y ecológicos de estos ambientes y sus posibles vías de contaminación.

Las concentraciones detectadas en el local pueden deberse a que en el momento del muestreo los acondicionadores de aire estaban averiados y por tanto las ventanas estaban abiertas. En el exterior de la Mapoteca se encuentra la Avenida del Puerto que se caracteriza por un alto nivel de movimiento vehicular además de la contaminación proveniente de la Terminal de Ferrocarril y las viviendas circundantes, es muy probable que estos hayan sido las fuentes causantes de estos valores de carga fúngica. Concentraciones similares han sido detectadas en Cuba en ambientes interiores de viviendas, archivos, bibliotecas y museos que han sido muestreados con biocolectores (Rojas, 1998; Rojas et al. , 2002; Pons y Rojas, 2003; Borrego y Perdomo, 2012; Rojas y Aira; 2012). Sin embargo, en estudios previos (Vaillant, et al 1989) realizados en otros depósitos del ARNAC utilizando un biocolector (aeroscopio), se encontraron concentraciones significativamente menores. Esto demuestra a) la necesidad de realizar muestreos sistemáticos para caracterizar la variabilidad de la micobiota, y b) que esa elevada contaminación del aire de los depósitos pudiera deberse a que el ambiente que rodea al ARNAC posee una elevada contaminación microbiana que se introduce en los depósitos a través de los conductos de ventilación natural (Borrego et al., 2008; Borrego et al., 2010).

Como es sabido, la humedad relativa del aire tiene gran importancia para la contaminación microbiana ambiental, sobre todo, en el caso de los hongos donde incide en el nivel de esporulación, en el diámetro aerodinámico de las esporas, así como en la deposición de éstas sobre las superficies e incluso en el sistema respiratorio de las personas (Florian, 2004; Borrego, 2005; Rojas y Aira; 2012). Teniendo en cuenta que la HR en la Mapoteca resultó de un $67.5 \%$ y que está por encima de lo recomendado para la conservación de mapas y planos (Walker 2003; Resolución No. 41/2009, CITMA) se puede afirmar que, las condiciones ambientales de este local facilitaron la presencia de elevadas concentraciones de hongos capaces de depositarse sobre las superficies, lo cual constituye un riesgo para el buen estado de conservación de los distintos tipos de documentos y para la salud del personal que habitualmente entra y sale de este local. 
Análisis ecológico de los hongos aislados del aire

Del aire de la Mapoteca se aislaron 27 colonias de hongos filamentosos diferentes, 26 fueron ubicadas en 9 géneros y una no esporuló en los medios evaluados. De todos los aislados identificados el $96 \%$ se clasificaron como anamorfos de ascomicetes (tabla II) correspondientes a los géneros Alternaria Micheli ex Fries, Aspergillus Nees ex Fr., Acremonium Link ex Fr, Cladosporium Link ex Fr., Curvularia Boedijn, Epicoccum Link, Penicillium Link, Pestalotia Not., y Trichoderma Pers. Los géneros antes mencionados se ubicaron en los órdenes Eurotiales, Pleosporales, Xylariales, Hypocreales y Capnodiales. Estos resultados concuerdan con estudios realizados por otros autores en ambientes de archivos y bibliotecas donde han predominado los representantes de dichos ordenes a exepción de los Xylariales (Valentín, 2001; Borrego et al., 2010 a; Borrego y Perdomo, 2012). Por ello cabe destacar que resulta un nuevo reporte para el aire de depósitos del ARNAC el género Pestalotia Not.

Es importante resaltar que estos resultados son característicos del método de muestreo empleado pues al utilizar placas con medios de cultivo, se desarrollan con facilidad las fases anamórficas de los hongos que sedimentan (Rojas, 2010).

\section{Densidades relativas de los géneros identificados en el aire}

De los 9 géneros fúngicos identificados, los de mayor DR (figura 1) en orden decreciente fueron Aspergillus, Penicillium, Curvularia y Pestalotia. Estos resultados concuerdan solo de forma parcial con los obtenidos por otros autores (Rojas, et al., 2008) incluso en estudios realizados años atrás en el mismo local (García, 1997; Guiamet et al., 2011). Muchos autores dentro de los que se incluyen los antes mencionados plantean que los géneros más represen- tativos en el aire tanto de interiores como de exteriores son Aspergillus, Penicillium y Cladosporium, mientras que en este estudio Curvularia sustituyó a Clasdosporium. Otros autores (Meklin et al., 2005; Lignell, 2008; Rojas, 2010; Rojas et al., 2012) han demostrado que los conidios de Aspergillus y Penicillium en general son más numerosos en el aire de interiores que de exteriores de lo que se deduce que muchos de ellos provienen de los materiales y soportes contaminados en dicho medio. Penicillium es el género más abundante en ambientes interiores de viviendas y Aspergillus es el más común en el aire de viviendas húmedas al igual que en archivos y almacenes soterrados seguido de Penicillium (Rojas, 2010). Es conveniente recordar que el muestreo se realizó en el mes de julio (mes que corresponde a la temporada lluviosa en Cuba) y que el local no estaba climatizado sino ventilado naturalmente lo que facilitó la existencia de valores de HR superiores al $60 \%$ que a su vez son elevados para la conservación de mapas y planos (Resolución No. 41/2009, CITMA). Resulta interesante además, el hecho de que se ha demostrado que los géneros Aspergillus y Penicillium son los más frecuentes en ambientes interiores en Cuba (Valliant et al., 1989; Fernández et al., 1990; Aira et al., 2002; Borrego et al., 2008, Borrego y Perdomo, 2012).

El género Curvularia ha sido detectado en ambientes interiores colonizando diferentes sustratos 0 en el aire (Borrego et al., 2008; Nevalainen y Morawska, 2009; Rojas 2010), por otra parte este hongo dematiáceo es típico de la atmósfera en regiones tropicales y subtropicales del mundo (Levetin y Dorsey, 2006; Rocha et al., 2013) incluso se ha reportado por Almaguer y Rojas (2013) como uno de los más abundantes en la atmósfera de La Habana, Cuba. El caso de Pestalotia no se encuentra reportado como un género común en ambientes interiores aunque si ha sido detectado en este nicho ecológico

Tabla II. Anamorfos de ascomicetes detectados en el aire, número de aislados y densidades relativas.

\begin{tabular}{|llcc|}
\hline Clasificación & Orden & No. de aislados & DR (\%) de los taxa \\
\hline Anamorfos de ascomicetes & Eurotiales & 52 & 53 \\
& Pleosporales & 19 & 19 \\
& Xylariales & 10 & 10 \\
& Hypocreales & 8 & 8 \\
& Capnodiales & 7 & 8 \\
\hline
\end{tabular}

DR (\%) densidad relativa con respecto al total de colonias de hongos obtenidas 


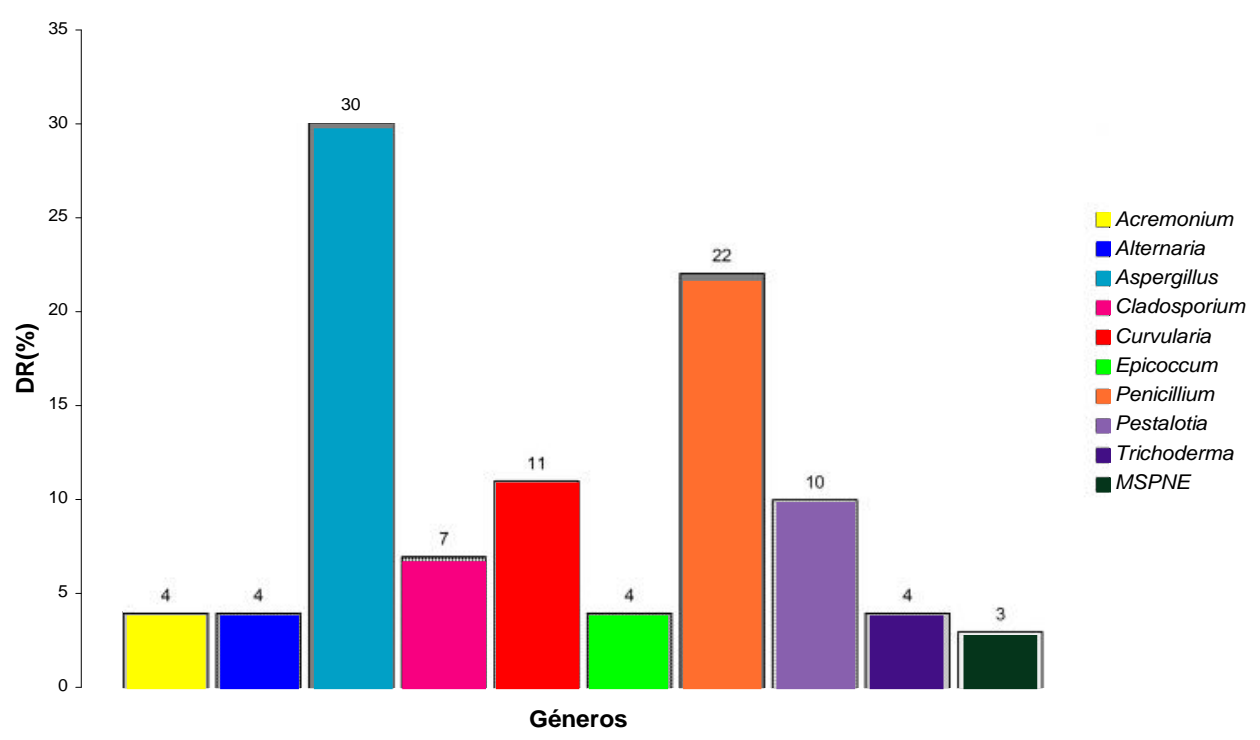

Figura 1. Densidad relativa (\%) de los géneros fúngicos aislados en el aire de la Mapoteca. MSPNE: Indica micelio septado pigmentado no esporulado.

(Samsom y Hoekstra, 1994); este hongo es un saprófito de plantas y típico de ambiente exteriores por lo que su aislamiento en el local estudiado pudiera deberse, al igual que Curvularia, a la penetración de sus propágulos por las ventanas del recinto el cual posee en su exterior abundante vegetación. La detección de ambos géneros evidencia la influencia que puede ejercer el ambiente exterior sobre el interior y además acentúa la importancia de realizar muestreos microbiológicos periódicos del aire, ecosistema cuya microbiota es alóctona y extremadamente dinámica. El resto de los géneros identificados presentaron un porcentaje de DR por debajo del 7\%, siendo Epicoccum (4\%) y Trichoderma (3\%) los que presentaron los valores más bajos. Los géneros encontrados con menor DR en ambientes interiores varían de acuerdo a los resultados obtenidos por otros autores (Valentín; 2003; Rojas, 2010; Borrego et al., 2010 a).

\section{Identificación específica de los hongos aislados del aire}

Se realizó la identificación hasta especie de los diferentes aislados y se calculó la densidad relativa de cada una de las especies dentro de su género (tabla III). De todas las especies identificadas destacaron algunas que se encuentran reportadas por varios autores ya sea por sus altas DR dentro del género, por su importancia como agentes biodeteriorantes 0 las afecciones que pueden causar a la salud. Dentro del género Aspergillus se identificaron el $100 \%$ de los aislados y como resultado se obtuvieron un total de 7 especies, entre ellas $A$. flavus Link., A. oryzae Ahlb Cahn. y A. ostianus Wehmer. resultaron ser las de mayor DR (23\%, 17\% y 17\%, respectivamente). Resultados similares han sido reportados en ambientes de bibliotecas y archivos (Rojas, 2010) incluyendo otros depósitos del ARNAC (Valliant et al., 1989; Borrego et al., 2008). A. flavus y A. oryzae han sido considerados de importancia para el biodeterioro, pues son capaces de crecer a expensas de una gran variedad de sustratos (Rojas, 2010), y para la salud humana (Klich, 2006; Amaike y Keller, 2011) al ser dos de los principales causantes de la aspergilosis bronquial alérgica y el primero de ellos además, de otitis e infecciones pulmonares (De Hoog et al; 2000). Le siguieron en orden descendente $A$. candidus Link. у A. japonicus Saito. ambos con DR del 13\%, A. alliaceus Thom y Church. con DR de $10 \%$ y $A$. auricomus (Gueren) Saito. con DR de 7\%; cabe señalar que $A$. ostianus, $A$. alliaceus y $A$. auricomus se obtuvieron por primera vez en estudios realizados en el ARNAC.

El 100\% de los aislados del aire del género Cladosporium correspondieron a representantes del complejo de especies C. cladosporioides (Fres.) de Vries., esto concuerda con otros estudios realizados en ambientes de bibliotecas y archivos (Valentín, 2001, 2004; Rojas, 2010; Borrego y Perdomo, 2012). Estas especies se encuentra reportadas por otros autores como las de mayor predominio dentro del género en ambientes interiores (Anderson, 2003; Payam y 
Tabla III. Identificación y DR de los hongos aislados de aire

\begin{tabular}{|c|c|c|}
\hline Hongos & No. colonias & DR $(\%)$ \\
\hline Acremonium sp. Link. & 4 & 100 \\
\hline Alternaria sp. Micheli ex Fries. & 4 & 100 \\
\hline Aspergillus alliaceus Thom y Church.* & 3 & 10 \\
\hline Aspergillus auricomus Saito.* & 2 & 7 \\
\hline Aspergillus candidus Link. & 4 & 13 \\
\hline Aspergillus flavus Link. & 7 & 23 \\
\hline Aspergillus japonicus Saito. & 4 & 13 \\
\hline Aspergillus oryzae Cohn. & 5 & 17 \\
\hline Aspergillus ostianus Whemer.* & 5 & 17 \\
\hline Cladosporium cladosporioides (Fres.) de Vries. (complex) & 7 & 100 \\
\hline Curvularia pallecens Boeding y Bull. & 5 & 45 \\
\hline Curvularia australiensis Manamgoda, Cai y Hyde.* & 4 & 36 \\
\hline Curvularia eragrostidis Henn. & 2 & 18 \\
\hline Epicoccum sp. Link. & 4 & 100 \\
\hline Penicillium canescens Sopp.* & 4 & 18 \\
\hline Penicillium chrysogenum Thom. & 6 & 27 \\
\hline Penicillium citrinum Thom. & 1 & 5 \\
\hline Penicillium sp. Link. 1 & 3 & 14 \\
\hline Penicillium sp. Link. 2 & 3 & 14 \\
\hline Penicillium sp. Link. 3 & 5 & 23 \\
\hline Pestalotia sp. Not. 1 & 8 & 80 \\
\hline Pestalotia sp. Not. 2 & 1 & 10 \\
\hline Pestalotia sp. Not. 3 & 1 & 10 \\
\hline Trichoderma sp. Pers. & 4 & 100 \\
\hline Micelio septado pigmentado no esporulado & 3 & 100 \\
\hline
\end{tabular}

* Indica que estas especies fueron detectados por primera vez en el aire de la Mapoteca del ARNAC.

Ramanathan, 2004; Borrego et al., 2008; Guiamet et al., 2011; Hung et al; 2011). Además representantes del complejo han sido detectados en interiores de viviendas con problemas de humedad en otros países como Bélgica (Beguin y Nolard, 1994). C. cladosporioides es asociado con infecciones cutáneas, oculares y nasales que pueden llegar a ser graves en pacientes inmunocomprometidos (Vaillant, 1996; Payam y Ramanathan, 2004; Haleem y Mohan, 2012). De las tres especies identificadas del género Penicillium, $P$. chrysogenum (27\%) y P. citrinum (5\%) coinciden con dos de las especies detectadas anteriormente por Borrego et al. (2008) y Borrego y Perdomo (2012) en estudios realizados en otros depósitos del ARNAC, además de otros estudios realizados en ambientes de archivos y bibliotecas cubanos por Rojas (2010). Esto pudiera indicar que ambas especies son típicas de este tipo de ambiente. Pueden jugar un papel importante en el biodeterioro de los soportes documentales, P. chrysogenum por su alto potencial para deteriorar materiales de origen celulósico (Villalba et al., 2004) y P. citrinum por su gran capacidad de producir pigmentos (Pitt, 2000). Por otra parte algunas cepas en ambos casos se han reportado como causantes de keratitis, endocarditis y en el caso de la segunda especie, además puede ocasionar infecciones del tracto urinario (De Hoog et al; 2000). La especie Penicillium canescens Sopp. que se detectó con una 
DR del $18 \%$ resulta ser un nuevo hallazgo para el ARNAC.

Otra especie fúngica detectada con alta DR fue Curvularia australiensis Manamgoda, Cai y Hyde, cuyo hallazgo constituyó una novedad en el ambiente de Ios depósitos del ARNAC aunque se ha detectado en otros estudios realizados en otros países (Nevalainen y Morawska, 2009). Este hongo dematiáceo se ha reportado como causante de casos de infecciones subcutáneas y sinusitis en humanos (De Hoog et al; 2000).

\section{Grado de contaminación fúngica en el polvo sedimentado}

Al analizar la concentración fúngica en el polvo aspirado en el interior de la Mapoteca se detectó una concentración media de $6 \times 10^{5}$ UFC. g $^{-1}$. No existe un estudio precedente realizado en el ARNAC de detección de hongos en el polvo. Sin embargo se reportan concentraciones de orden similar $\left(10^{5}\right)$ por otros autores en estudios realizados a partir del polvo casero en otros países (Engelhart et al., 2002; Chew et al., 2003; Heinrich et al., 2003; Horner et al., 2004; Hicks et al., 2005; Lignell, 2008). Incluso se plantea que la concentración fúngica puede variar de $6 \times 10^{3}$ hasta $3.2 \times 10^{6} \mathrm{UFC} . \mathrm{g}^{-1}$ de polvo (Canhoto et al., 2004). El polvo es considerado por varios autores como una fuente importante de información en estudios de diagnóstico microbiológico en un ambiente interior (Portnoy et al, 2004; Schram-Bijkerk et al., 2006; Lignell, 2008), ya que constituye un reservorio de la contaminación microbiana y en especial de hongos provenientes del aire. Nevalainen y Morawska (2009) lo reafirman en su Documento Guía (OMS), donde además reúnen los datos de géneros y especies fúngicas detectadas en una gran cantidad de estudios microbiológicos realizados en este nicho ecológico por varios autores de gran parte del planeta, llegando a reportarse más de 20 géneros y 200 especies. De esta forma, el polvo por una parte, constituye un peligro potencial para las colecciones de valor patrimonial (Portnoy et al., 2004) al hidratarse, cuando la HR aumenta, propiciando las condiciones para que los hongos puedan desarrollarse y establecerse sobre él o sobre los soportes y por otro lado, constituye un reservorio de alergenos, esporas, microorganismos y otros elementos que pueden ser perjudiciales para la salud.

Análisis ecológico de los hongos aislados del polvo Del polvo aspirado en la Mapoteca se aislaron doce colonias de hongos filamentosos diferentes, el $87 \%$ de los aislados se clasificaron como anamorfos de ascomicetes (tabla IV). Los aislados antes mencionados se ubicaron en los órdenes Eurotiales, Capnodiales y Sordadiales, correspondieron a representantes de los géneros Aspergillus, Cladosporium, Humicola Traaen y Penicillium. El 13\% restante fueron holomorfos de ascomicetes que se clasificaron como Sordadiales y Eurotiales de los géneros Chaetomium Kunze ex Fr. y Aspergillus. Aunque se evidenció el predominio de los hongos mitospóricos en el polvo (87\%) se puede apreciar una disminución de su DR con respecto al ecosistema aéreo. Este resultado está condicionado en gran parte por el medio hipertónico que pudieron imponer las partículas de polvo como parte del sustrato a los propágulos fúngicos, que actúa como una barrera selectiva para la germinación y el desarrollo de las esporas y otras estructuras. Además, acentuó este fenómeno la presencia de sales marinas en el polvo que sedimenta en la Mapoteca ya que el ARNAC se encuentra ubicado a $174 \mathrm{~m}$ del mar y se han detectado

Tabla IV. Anamorfos y holomorfos de ascomicetes detectados en el polvo, número de aislados y densidades relativas.

\begin{tabular}{|llcc|}
\hline Clasificación & Orden & No. de aislados & DR (\%) de los taxa \\
\hline \multirow{2}{*}{ Anamorfos de ascomicetes } & Eurotiales & 101 & 64 \\
\cline { 2 - 4 } & Capnodiales & 33 & 20 \\
\cline { 2 - 4 } & Sordadiales & 5 & 3 \\
\hline \multirow{2}{*}{ Holomorfos de ascomicetes } & Eurotiales & 16 & 10 \\
\cline { 2 - 4 } & Sordadiales & 4 & 3 \\
\hline
\end{tabular}

DR (\%) densidad relativa con respecto al total de colonias de hongos obtenidas 
concentraciones considerables de iones cloruro en su composición (equivalentes a un promedio de $5.6 \mathrm{x}$ $10^{-5} \mathrm{mg}$ de iones cloruros por $\mathrm{mg}$ de polvo, lo que equivale al $2.8 \%$ de las sustancias solubles en el polvo) (Vivar y Borrego, 2012). Se ha reportado que cantidades de sales entre un 3 y un $4 \%$ sobre el papel propician la presencia de hongos xerófilos como son los representantes del género Aspergillus (Michaelsen et al., 2010).

\section{Densidades relativas de los géneros identificados en el polvo}

De los 5 géneros fúngicos aislados del polvo aspirado los de mayor $\mathrm{DR}$ en orden decreciente fueron Aspergillus, Penicillium y Cladosporium (figura 2). Estos resultados coinciden de forma parcial con los obtenidos por otros autores (Chao et al, 2002; Lignell, 2008; Haleem y Mohan, 2012) siendo estos tres géneros siempre los predominantes en el polvo colectado, pero variando sus densidades relativas en Ios diferentes estudios. El polvo como ya se ha mencionado puede ser un reservorio de propágulos fúngicos y puede constituir un referente para el estudio del comportamiento de la micobiota aérea de toda una temporada (Portnoy et al., 2004; Pasquarella et al., 2012). En este caso, el polvo colectado representa la acumulación de propágulos de una semana. Al comparar estos resultados con los obtenidos en el muestreo del aire se aprecia similitud en cuanto a los géneros con mayores DR ubicándose Aspergillus y Penicillium como los dos primeros en ambos casos. El predominio de los géneros Aspergillus y Penicillium y la detección de representantes del género Cladosporium demuestran la relación existente entre la micobiota presente en el aire y en el polvo sedimentado

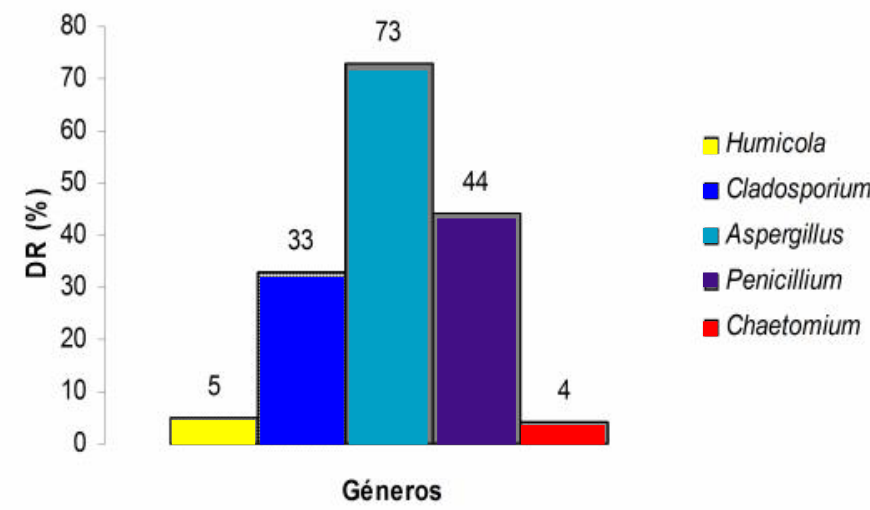

Figura 2. Densidad relativa (\%) de los géneros fúngicos aislados del polvo aspirado en la Mapoteca. en el local estudiado. Los propágulos fúngicos en el aire siguen la dinámica de los bioaerosoles atmosféricos (Leventin, 2002; Chin y Heinsohn, 2007). Este resultado lo respalda la relación entre las fases de deposición y resuspensión de las partículas biológicas, dichas fases pueden sucederse de forma cíclica en interiores producto de la climatización o las corrientes de aire. Por lo antes expuesto, se demuestra que existe la necesidad de realizar muestreos en ambos microambientes (aire y polvo sedimentado) a la hora de caracterizar un ambiente interior (Pasquarella et al., 2012).

Los tres géneros restantes identificados mostraron una DR por debajo del $10 \%$ siendo Humicola (3\%) el de menor valor, este último es un género típico del humus del suelo que no se encuentra con frecuencia en interiores, pero que de establecerse sobre los soportes documentales traería graves consecuencias por ser altamente celulolítico (Domsch et al., 1980). Al comparar con otros resultados consultados se observó variación en la diversidad de géneros obtenidos para los valores menores de DR (Chao et al, 2002; Chew et al., 2003; Yang y Li, 2007; Lignell, 2008). Es interesante el hallazgo de holomorfos de Aspergillus ssp. en diversos estudios del polvo casero (Lignell, 2008; Michaelsen et al., 2010; Haleem y Mohan, 2012).

\section{Identificación específica de los hongos aislados del polvo}

Al igual que en el caso de los aislados del aire se calculó la DR de los géneros y especies (tabla $\mathbf{V}$ ). De todas las especies identificadas destacaron algunas por diversas razones, como haber sido aisladas también en el muestreo aéreo realizado en este estudio, por encontrarse reportadas por varios autores, ya sea por sus altas DR dentro de su género en ambientes de archivos y bibliotecas, por su importancia como agentes biodeteriorantes o ser causantes de afectaciones a la salud humana.

Dentro del género Aspergillus se identificaron el $100 \%$ de los aislados detectándose representantes del complejo de especies A. niger y A. flavus con DR de 27 y $50 \%$, respectivamente. Estos resultados coinciden con los obtenidos por Chao et al (2002) y Lignell (2008) en aislados de polvo colectado en un ambiente interior. Al comparar con los resultados obtenidos en el muestreo aéreo (tabla III) coincide 
Tabla V. Hongos aislados del polvo depositado sobre el mobiliario (planeros) de la Mapoteca.

\begin{tabular}{|lll|}
\hline Hongos & No. colonias & DR \% \\
\hline Aspergillus flavus Link. & 36 & 50 \\
Aspergillus chevalieri Mangin Thom. Y Church. ${ }^{*}$ & 16 & 23 \\
Aspergillus niger Batista, Maia y Alecrim. (complex) & 21 & 27 \\
Chaetomium globosum. Kunze & 4 & 100 \\
Cladosporium caryigenum Ellis y Lang. ${ }^{*}$ & 12 & 36 \\
Cladosporium cladosporioides (Fres.) de Vries. (complex) & 21 & 64 \\
Humicola sp. Traaen. & 5 & 100 \\
Penicillium janczewskii K.M. Zalessky. ${ }^{*}$ & 19 & 43 \\
Penicillium sp.4 Link. & 8 & 18 \\
Penicillium sp.5 & 17 & 39 \\
\hline
\end{tabular}

* Indica que estas especies fueron detectados por primera vez en la Mapoteca del ARNAC.

que $A$. flavus es la especie de mayor DR dentro del género. Este resultado es un indicativo de la prevalencia de la especie dentro del local estudiado, lo cual constituye un elemento importante a tener en cuenta tanto para la salud del personal como para el biodeterioro de los soportes.

A. flavus junto a $A$. fumigatus pertenecen a un grupo de hongos que se encuentran reportados como peligrosos para la salud humana (Denning, 2006). Al ser hongos que resisten condiciones ambientales hostiles y tener la capacidad de comportarse como oportunistas, se propone para ellos la clasificación de seguridad biológica grado 2 (Nivel de seguridad biológica, BSL, según sus siglas en inglés) (Hoog, 1996), a diferencia de la mayoría de las especies de hongos encontradas en ambientes interiores que clasifican para un BSL-1 (Rojas, 2010). En el caso de las especies del complejo $A$. niger igualmente han sido consideradas de importancia para la salud humana, además están reportadas como predominantes en el país en ambientes de archivos y bibliotecas incluyendo depósitos del ARNAC (Klich, 2006; Rojas, 2010; Borrego et al., 2008; Borrego y Perdomo, 2012). Los dos holomorfos detectados del género Aspergillus resultaron ser Aspergillus chevalieri Mangin. El cual ha sido reportado en estudios realizados en ambientes interiores en Cuba (Rojas, 2010) y más específicamente fuera de Cuba en aislamientos a partir del polvo (Meklin et al., 2004; Vesper et al., 2007). La presencia de esta especie en el polvo de la Mapoteca pudiera deberse a las características xerófilas de la misma (Klich y Pitt, 1994) que encuentra en el polvo niveles de sequedad idóneos para el mantenimiento de su viabilidad. Igualmente, esta constituye el primer reporte para el ARNAC.

De los aislados del género Cladosporium se detectaron especies del complejo C. cladosporioides y C. caryigenum Ellis y Lang. En cuanto a la primera, coinciden los resultados obtenidos por otros autores como Lignell (2008) y Yang y Li (2007) que reportaron su detección en polvo con altos valores de DR. Dicho resultado pudiera deberse a que algunas cepas se consideran ligeramente xerófilas pues se caracterizan por su capacidad de soportar determinados niveles de sequedad 0 xerofilia (Payam y Ramanathan, 2004), condición que encuentran en el polvo; aunque de manera general los representantes del género requieren de humedad para crecer (Nielsen 2003; Górny 2004). Esta cepa además de encontrarse en el polvo sedimentado, fue la única de su género detectada en el muestreo aéreo, resultado que indica la prevalencia de la misma dentro de la Mapoteca. $C$. cladosporioides generalmente es asociado con infecciones cutáneas, oculares y nasales (Vaillant, 1996; Haleem y Mohan, 2012), se considera además conjuntamente con otras especies un alérgeno importante pues los conidios y fragmentos de hifas son capaces de provocar estados alérgicos del Tipo I 
(asma y fiebre del heno) y del Tipo III (neumonía por hipersensibilidad) (Abbott, 2002), de ahí que se plantee que aproximadamente el $10 \%$ de la población es sensible a este hongo. Es de destacar que la especie C. caryigenum constituye un nuevo reporte para ambientes de depósitos del ARNAC. El hallazgo de $C$. cladosporioides y Aspergillus flavus en aire y polvo en este estudio es indicativo de su predominio en este ecosistema.

Dentro de las especies del género Penicillium, se detectó una correspondiente a $P$. janczewskii K.M. Zalessky con una DR del $41 \%$, que se considera alta para este nuevo reporte en el ARNAC.

\section{CONCLUSIONES}

El ambiente de la Mapoteca del Archivo Nacional de la República de Cuba presentó un valor promedio de concentración microbiana inferior a las 750 UFC.m${ }^{3}$ de hongos según la escala de Omeliansky, por tanto el local se consideró poco contaminado. La concentración de propágulos fúngicos viables en el polvo sedimentado sobre el mobiliario (planeros) de la Mapoteca fue de $6.10^{5}$ UFC. ${ }^{-1}$, lo cual evidencia que el polvo puede constituir un reservorio de la micobiota en ambientes interiores. En el ambiente interior del depósito se identificaron nueve géneros y trece especies, mientras que en el polvo fueron identificados cinco géneros y cinco especies. Esto constituye la caracterización fúngica más integral realizada en el Archivo Nacional de la República de Cuba, lo que incrementa el conocimiento de la biodiversidad de este tipo de ambiente. El hallazgo de C. cladosporioides y Aspergillus flavus en aire y polvo es indicativo de su predominio en este ecosistema. Constituyeron nuevos registros para este archivo cubano los géneros, Epicoccum Link, Humicola Traaen y Pestalotia Not. y las especies Aspergillus ostianus Wehmer., A. alliaceus Thom y Church., A. auricomus (Gueren) Saito, Curvularia australiensis Manamgoda, Cai y Hyde., C. caryigenum Ellis y Lang., Aspergillus chevalieri Mangin Thom. y Church., Penicillium canescens Sopp. y P. janczewskii K.M. Zalessky.

\section{AGRADECIMIENTOS}

Los autores agradecen el financiamiento recibido por el Programa de Ayuda a los Archivos de Iberoamérica (ADAl) a través de los Proyecto 146/2008 y 134/2010.

\section{REFERENCIAS}

Abbott S.P. (2002). Molds and other fungi in indoor environments. Summary of biology, known health effects and references. [en línea] http://www.precisionenv.com/ PDFS/IndoorMolds1.pdf 30/09/2003.

Aira M. J., Rojas T. y Jato V. (2002). Fungi associated with three houses in Havana (Cuba). Grana 41, 114-118.

Almaguer M. y Roja T.I. (2013). Aerobicota viable de la atmósfera de La Habana, Cuba. Nova Acta Científica Compostelana (Bioloxía). 20, 35-45.

Amaike A. y Keller N.P. (2011). Aspergillus flavus. Annu. Rev. Phytopathol. 49, 10.1-10.27.

Anderson J.B. (2003). Heritage, conservation \& health - the Danish experience. European Heritage Conservation. Environmentally Sustainable Solutions, Trinity College, Dublin, Ireland. 25 - 26 September, 2003. [en línea] http://www.tkbassociates.com/seminars/isbe\%20presentations/ JorgenBechAndersonISBEPaper.doc 02/11/2004.

Barnett, H.L. and B.B. Hunter. 2003. Illustrated genera of Imperfect fungi. 4th ed. Burgués. Publishing Company Minneapolis 15, Minn.

Beguin H. y Nolard N. (1994). Mould biodiversity in homes, I. Air and surface analysis of 130 dwellings. Aerobiologia. 10,157-166

Bogomolova E.V. y Kirtsideli I. (2009). Airborne fungi in four stations of the St. Petersburg underground railway system. Int. Biodeter. Biodegr. 63, 156-160.

Borrego S. y Perdomo I. (2012). Aerobiological investigations inside repositories of the National Archive of the Republic of Cuba. Aerobiologia. 28, 303-316

Borrego S., Guiamet P., Gómez de Saravia S., Battistoni P., García M., Lavín P. y Perdomo I. (2010 a). The quality of air at archives and the biodeterioration of photographs. Int. Biodeter. Biodegr. 64, 139-145.

Borrego S., Perdomo I., Guiamet P. y Gómez S. (2010). Estudio de la concentración microbiana en el aire de los depósitos del Archivo Nacional de Cuba. 
AUGMDOMUS 1, 114-133.

Borrego S., Pons V. y Perdomo I. (2008). La contaminación microbiana del aire en dos depósitos del Archivo Nacional de la República de Cuba. Revista CENIC Ciencias Biológicas. 39, 63-69.

Borrego S. (2005). El edificio de archivo: su influencia en la contaminación microbiana ambiental, el biodeterioro y la salud del personal. IV Coloquio Iberoamericano del Papiro a la Realidad Virtual, Casa de las Américas, La Habana, Cuba, 21 al 25 de marzo, 2005.

Canhoto 0., Pinzari F., Fanelli C. y Magan N. (2004). Application of electronic hose technology for the detection of fungical contamination in library paper. Int. Biodeter. Biodegr. 54, 300-309.

Cappitelli F. y Sorlini C. (2010). Paper and manuscripts. En: Cultural heritage microbiology: Studies in Conservation Science. Mitchell R. y McNamara C.J. (Eds.). ASM Press, Washington, DC, 45-59.

Cappitelli F., Fermo P., Vecchi R., Piazzalunga A., Valli G., Zanardini E. y Sorlini C. (2009). Chemicalphysical and microbiological measurements for indoor air quality assessment at the $\mathrm{Ca}$ 'Granada Historical Archive, Milan (Italy). Water Air Soil Poll. 201, 109-120.

Casadesús L.y Rojas T.I. (1981). Micología. Manual Práctico. Ed. MES; La Habana. 99 pp.

Castañeda R.F., Fabré D.E., Parra M.P.P., Pérez M., Guarro J. y Cano J. (1996). Some airborne conidial fungi from Cuba. Mycotaxon. 60, 283- 290.

Chao H.J., Milton D.K., Schwartz J. y Burge A. B. (2002). Dustborne fungi in large office buildings. Mycopathol. 154, 93-106.

Chew G. L., Rogers C., Burge H. A., Muilenberg M. L. y Gold D. R. (2003). Dustborne and airborne fungal propagules represent a different spectrum of fungi with differing relations to home characteristics. Allergy $58,13-20$.

Chin S.Y. and Heinsohn. P. (eds.) (2007). Sampling and Análisis of Indoor Microorganisms. Jhon Wiley \& Sons, Inc., Hoboken, New Jersey.263pp.

De Hoog, G.S., Guarro, G., Gene, J. Figueras, M.J. (2000). Atlas of clinical fungi. $2^{\text {nd }}$ ed. (Eds): Deiana, P.,
Fatichenti, F., Farris, G.A., Mocquot, G., Lodi, R., Todesco, R. and Cecchi. Universidad Rovira I Virgili Reus, España

Denning D.W. (2006). Aspergilosis. Schering-plough Corporation. 76 pp.

Domsch K.H., Gams W., Anders T.H. (eds.) (1980). Compendium of Soil Fungi. Institute of Soil Biology Federal Agricultural Research Centre Braunschweig. Germany, vol.1.pp 393-397.

Engelhart S., Loock A., Skutlarek D., Sagunski H., Lommel A., Färber H. y Exner M. (2002). Occurrence of toxigenic Aspergillus versicolor isolates and sterigmatocystin in carpet dust from damp indoor environments. App. Environ. Microb. 68, 3886-3890.

Fernández S., Sánchez A., Cabezas A., Medina T., Aguilar A. y Novoa, G. (1990). Estudio preliminar del grado de contaminación biológica en almacenes soterrados que provocan el biodeterioro de diferentes materiales. Quimindustria'90. Memorias del I Simposio Internacional de Corrosión-Protección y Tropicalización, 232-235.

Florian M.L.E. (2004). Fungal facts. Solving fungal problems in heritage collections. Archetype Publications Ltd., London, UK, 146 pp.

Florian M.L.E. (2003). The four components of biodeterioration and of preservation of our collective memory. International Symposium: a choice and strategies for preservation of a collective memory. Dobbiaco, Toblach, Italy. [en línea] http://www.uinmuenster.de/Forum-Bestandserhaltung/konsrestaurierung/sch florian.shtml 05/10/2002.

García T. (1997). Consideraciones sobre la microbiota de la Mapoteca del Archivo Nacional. Tesis de Licenciatura. Facultad de Biología. Universidad de la Habana. La Habana, Cuba.

Górny R.L. (2004). Filamentous microorganisms and their fragments in indoor air: A review. Agr. Environ. Med. 11, 85-197.

Guiamet P., Crespo M., Lavin P., Ponce B., Gaylarde C. y Gomez de Saravia S. (2013). Biodeterioration of funeral sculptures in La Recoleta Cemetery, Buenos Aires, Argentina: Pre- and post-intervention studies. Colloid Surface B. 101, 337-342. 
Guiamet P., Rosato V., Gómez de Saravia S., García A.M. y Moreno D.A. (2012). Biofouling of crypts of historical and architectural interest at La Plata Cemetery (Argentina). J. Cult. Herit. 13, 339-344.

Guiamet P., Borrego S., Lavin P., Perdomo I. y Gómez de Saravia S. (2011). Biofouling and biodeterioration in materials stored at the Historical Archive of the Museum of La Plata, Argentine and at the National Archive of the Republic of Cuba. Colloid Surface B. 85, 229-234.

Haleem A.A. y Mohan S. (2012). Fungal pollution of indoor environments and its management. Saudi J. Biol. Sci. 19, 405-426

Heinrich J., Hölscher B., Douwes J., Richter K., Koch A., Bischof W., Fahlbusch B., Kinne R.W., Wichmann H.E. (2003): Reproducibility of allergen, endotoxin and fungi measurements in the indoor environment. J. Expo. Anal. Env. Epid. 13, 152-160.

Hicks J. B., Lu E. T., De Guzman R. y Weingart M. (2005). Fungal types and concentrations from settled dust in normal residences. J. Occup. Environ. Hyg. 2, 481-492.

Hoog G.S. (1996). Risk assessment of fungi reported from humans and animals. Diagnosis, Therapy and Prophylaxis of fungal diseases. Mycoses 39, 407-417.

Horner W.E., Worthan A.G. y Morey P.R. (2004). Air and dustborne mycoflora in houses free of water damage and fungal growth. App. Environ. Microb. 70, 6394-6400.

Hung W., Su S.L., Shin L.Y., Roam G.D. y Chang T.C. (2011). Rapid, identification of allergenic and pathogenic molds in environmental air by an oligonucleotide array. BMC Infect. Dis. 11, 91 Dol: 10-1186/1471-2334-1191.

Huttunen K., Hyvärinen A., Nevalainen A., Komulainen H. y Hirvönen M.R. (2003). More intense production of proinflammatory mediators by indoor air bacteria than fungal spores in mouse and human cell lines. Environ. Health Persp. 111, 85-92.

InspectaPedia. Mould Exposure Standards. (2009). Levels of allergenic or toxic mould \& how much mould means a problem. Building and Environmental Inspection, Testing, Diagnosis, Repair, Problem Prevention Advice. [en línea] http://InspectaPedia.com/ sickhouse/Mold_Standards.htm 14/01/2010.
Johnston A. y Booth C. (1983). Plant Pathologist's Pocketbook. 2nd Ed. Commonwealth Mycological Institute. Kew, 439 pp.

Karbowska-Berent J., Górny R.L., Strzelczyk A.B. y Wlaz ${ }^{3}$ A A. (2011) Airborne and dust borne microorganisms in selected Polish libraries and archives. Build. Environ. 46, 1872-1879.

Kendrick, B. W., Carmichael, J. W. (1973) Hyphomycetes. Chapter 10. The Fungi. An advanced Treatise. Volume IVA. (Ed). G.C. Ainsworth. Institute Kew, Surrey, England.

Klich M. (2006). Identification of clinically relevant aspergilli. Med. Mycol. 44, 5127-5131.

Klich M.A. y Pitt J.I. (1994). A Laboratory guide to the common Aspergillus species and their teleomorphs. Commonwealth Scientific and Industrial Research Organization. $116 \mathrm{pp}$.

Levetin E. y Dorsey (2006). Contribution of leaf surface fungi to the air spora. Aerobiologia. 22, 3-12.

Levetin E. Bioaerosols in agricultural and out door setting. In: Encyclopedia of Environmental Microbiology. Bitton G. (Ed.). John Wiley and Sons, NY, 2002.

Lignell U. (2008). Characterization of microorganisms in indoor environments. Publications of the National Public Health Institute, ISSN 0359-3584; 1458-6290 (pdf-version). [en línea] http://www.ktl.fi/portal/4043 02/04/2009.

Maggi 0., Persiani A.M., Gallo F., Valenti P. y Pasquariello G. (2000). Airborne fungal spores in dust present in archives: proposal for a detection method, new for archival materials. Aerobiologia. 16, 429-434.

Mandrioli P. (2002). Bioaerosol and Biodeterioration. EC Advance Study Course 8-19 April. Science and Technology for Sustainable Protection of Cultural Heritage. Technical Notes for Session 7-8. UCL Center for Sustainable Heritage London, UK, 15 pp.

Mamangoda D.S., Cai L., Mckenzie E.H., Crous P.W., Madrid H., Chukeatirote E., Shivas R.G., Tan Y.P., Hyde K.D. (2013) A phylogenetic and taxonomic reevaluation of the Bipolaris - Cochliobolus - Curvularia 
Complex. Fungal Diversity. 56, 131-144.

Meklin T., Putus T., Pekkanen J., Hyvärinen A., Hirvonen M.R. y Nevalainen A. (2005). Effects of moisture-damage repairs on microbial exposure and symptoms in schoolchildren. Indoor Air 15, 40-47.

Meklin T., Haugland R.A., Reponen T., Varma M., Lummus Z., Bernstein D., Wymer L.J. y Vesper, S.J. (2004). Quantitative PCR analysis of house dust can reveal abnormal mold conditions. J. Environ. Monitor. 6, 615-620.

Mena J. (2004). Taxonomía del complejo Bipolaris, Curvularia, Drechlera y Exserohilum en Cuba. Tesis en opción al Grado Científico de Doctor en Ciencias Biológicas. Instituto de Ecología y sistemática, Ministerio de Ciencia Tecnología y Medio Ambiente (CITMA), La Habana, Cuba.

Michaelsen A., Piñar G. y Pizari F. (2010). Molecular and microscopical investigation of the microflora inhabiting a deteriorated Italian manuscript dated from the Thirteenth Century. Microb. Ecol. 60, 69- 80.

Nevalainen A. y Morawska L. (eds). (2009). Biological Agents in Indoor Environments. Assessment of Health Risks. Work conducted by a WHO Expert Group between 2000-2003. [en línea] http://www.laqh.qut.edu.au/Misc/ BIOLOGICAL_AGENTS_2009.pdf 06/09/2010.

Nielsen K.F. (2003). Mycotoxin production by indoor molds. Fungal Genet. Biol. 39, 103 - 117.

Pasquarella C., Saccani E., Sansebastiano G.E., Ugolotti M., Pasquariello G.Y Albertini R. (2012). Proposal for a biological environmental monitoring approach to be used in libraries and archives. Ann. Agr. Environ. Med. 19, 209-212.

Payam F. y Ramanathan K. (2004). Fungus of the month: Cladosporium species. The Environmental Report EMLab, Gallup, D. (Chairman), vol. 2, No. 4. [en línea] http://www.emlab.com 18/05/2006.

Pitt J.I. (2000). A laboratory guide to common Penicillium species. Third Ed. Food Science Australia. North Ryde, New South Wales, 196 pp.

Pons V. y Rojas T.I. (2003). Microbiota contaminante en el Museo Antropológico Montané. Tesis de Licenciatura, Facultad de Biología, Universidad de la Habana, Cuba.
Portnoy J.M., Barnes C.S. y Kennedy K. (2004). Sampling for indoor fungi. J. Allergy Clin. Immunol. 113, 189-198.

Portnoy J.M., Kwak K., Dowling P., Vanosdol T. y Barnes C. (2005). Health effects of in-door fungi. Ann. Allerg. Asthma Im. 94, 313-320.

Radler de Aquino F. y de Góes L.F. (2000). Guidelines for indoor air quality in offices in Brazil. Proc. Healthy Buildings. 4, 549-553.

Resolución No. 41 (2009). Lineamientos para la conservación de las fuentes documentales. Ministerio de Ciencia Tecnología y Medio Ambiente (CITMA), Gaceta oficial 9 de mayo 2009. [en línea] http:// www.gacetaoficial.cu/pp.193 11/05/2009.

Rojas T.I., Aira M.J., Batista A. y Cruz I.L. (2012). Fungal biodeterioration in historic buildings of Havana (Cuba). Grana 51, 44-51.

Rojas T.I. y Aira M.J. (2012). Fungal biodiversity in indoor environments in Havana, Cuba. Aerobiologia. 28, 367-374.

Rojas T.I. (2010). Diversidad fúngica en ambientes exteriores de áreas urbanas de ciudad de La Habana y sus potencialidades en el biodeterioro. Tesis en opción al Grado Científico de Doctor en Ciencias Biológicas. Facultad de Biología. Universidad de la Habana, La Habana, Cuba.

Rojas T.I., Martínez E., Aira M.J. y Almaguer M. (2008). Aeromicota de ambientes internos: Comparación de métodos de muestreo. Boletín Micológico 23, 67-73.

Rojas T.I., Martínez E., Gómez Y. y Alvarado Y. (2002). Airborne spores of Aspergillus species in cultural institutions at Havana University. Grana 41, 190e-193e.

Rojas T.I. (1998). Microbiota contaminante en ambientes interiores. Tesis en opción al Grado Científico de Maestro en Ciencias Biológicas, Facultad de Biología, Universidad de la Habana, La Habana, Cuba.

Samson, R. A. and Hoekstra, E. S. 1994. Common fungi occurring in indoor environments. In Health Implications Of Fungi In Indoor Environments (eds. R.A. Samson et al.), Elsevier, Amsterdam, pp. 541-587.

Sánchis J. (2002). Los nueve parámetros más críticos en el muestreo microbiológico del aire. Rev. Tecn. Lab. 
276: 858- 862.

SBM. (2003). Standard of Building Biology Testing Methods and Guidelines, Baubiologie, Maes/BNN.

Schram-Bijkerk D., Doekes G., Boeve M., Douwes J., Riedler J., Üblagger E., von Mutius E., Benz M., Pershagen G., Wickman M., Alfvén T., BraunFahrländer C., Waser M. y Brunekreef B. (2006). Exposure to microbial components and allergens in population studies: a comparison of two house dust collection methods applied by participants and fieldworkers. Indoor Air 16, 414-425.

Smith G. (1980). Ecology and Field Biology. 2nd Edition. Harper \& Row (eds.), New York.

Vaillant M. (1996). A work aimed to Project the health of the documental heritage conservators. International Conference on Conservation and Restoration of Archive and Library Materials. Pre-prints. Erice. 2229 April, 137-142.

Vaillant M., Chi L. y Sánchez, A. (1989). Sobre la contaminación microbiológica en depósitos del Archivo Nacional. Documentos 2, 44-62.

Valentín N. (2004). El biodeterioro de materiales orgánicos. Jornadas Monográficas Prevención del biodeterioro en archivos y bibliotecas. Instituto del Patrimonio Histórico Español. 14-15 junio, 2004, 84-89.

Valentin N. (2003). Microbial contamination and insect infestation in organic materials. COALITION 6, 2- 5.

Valentín N., García R., Ibáñez J.L. y Maekawa S. (2001). Tratamientos con ventilación controlada para detener el crecimiento microbiano en materiales de archivo. Archivamos. Ed. Asociación de Archiveros de Castilla y León (ACAL), № 39-40, 40-45.

Valentín N. (2001). Microbial contamination and insect infestation in Spanish museums and libraries. COALITION 3, 5-7.

Vesper S., McKinstry C., Ashley P., Haugland R., Yeatts K., Bradham K. y Svendsen E. (2007). Quantitative PCR analysis of molds in the dust from homes of asthmatic children in North Carolina. J. Environ. Monitor. 9, 826-830.

Villalba S., Mikán J.F. y Sánchez J. (2004). Actividades hidrolíticas y caracterización isoenzimáticas de poblaciones microbianas aisladas del patrimonio documental en el Archivo General de Colombia. Nova Publicación Científica 2, 50-57.

Vivar I. y Borrego S. (2012). Estudio del polvo sedimentado en depósitos del Archivo Nacional. Boletín del Archivo Nacional No. 18, 19, 20. [en línea] http://www.arnac.cu/wp-content/uploads/2013/03/ Estudio-del-polvo-sedimentado.pdf 15/05/2013.

Walter A. (2003). Basic preservation guidelines for library and archive collections. National Preservation Office, NPO Preservation Guidance, British Library. [en línea] http://www.bl.uk/b1pac/pdf/basic.pdf 09/02/ 2006.

Yang C.S. y Li D.W. (2007). Ecology of fungi in the indoor Environment. Cap.10 In: Yang C.S., Heinsohn P.A. (eds.) Sampling and analysis of indoor microorganism. Jhon Wiley and Sons, Inc., Hoboken, New Jersey, 263 pp. 